Discussion/conclusion As the trend of chemsex and sex parties continues, it is likely there will be an increase in STIs linked to households. Better geospatial analysis of STI trends and collaborative working with public health is essential for rapid identification and control of outbreaks.

\section{MSM REPORT HIGH USE OF CLUB DRUGS WHICH IS ASSOCIATED WITH HIGH RISK SEXUAL BEHAVIOUR}

${ }^{1}$ Thomas Kurka*, ${ }^{2}$ Suneeta Soni, ${ }^{2}$ Daniel Richardson. 'Brighton and Sussex Medical School, Brighton, UK; ${ }^{2}$ Brighton and Sussex University Hospitals NHS Trust, Brighton, UK

\subsection{6/sextrans-2015-052126.10}

Background/introduction The prevalence of club drug use in men who have sex with men (MSM) locally is unknown but likely associated with poor sexual health. Locally there is a large MSM population with high rates of HIV and STIs.

Aim(s)/objectives The aims of this study were to quantify club drug use in MSM locally, examine differences by HIV status and identify any association between club drug use and sexual behaviour.

Methods Patient survey of MSM attending three MSM-services (STI clinic, NGO, primary care centre) in the City. We asked MSM to report ever and recent (past month) drug use. Data were analysed using SPSS.

Results 246 MSM completed surveys from January-March 2014. The median age was 35 years (18-79). 12.7\% were HIVpositive, $61.1 \%$ HIV-negative, $20.0 \%$ unsure and $5.7 \%$ never tested. The overall ever: recent club drug use was: 52.4\%:21.5\% cocaine, 49.4\%:17.1\% MDMA, 37.7\%:19.3\% mephedrone, 35.5\%:10.5\% ketamine, 24.2\%:11.0\% GHB/GBL, and 10.4\%:2.8\% crystal meth. HIV-positive MSM reported significantly higher crystal meth (Ever:37.0\% v 6.9\%: p < 0.05; Recent 13.6\%:1.3\%: p < 0.05) and GHB/GBL (Ever:48.1\% v 21.2\%: p < 0.05; Recent: 27.3\%:8.9\%: p < 0.05) use than HIV-negative/unknown. HIV-positive were significantly more likely to have injected (Slamming) club drugs ever than HIV-negative/unknown (Ever: 22.2\% v 2.5\%: p < 0.05). HIV-positive MSM using club drugs reported significantly higher rates of unprotected anal intercourse (in past 6-months) than HIV-negative/unknown (87.1\% v 57.1\%: p < 0.05).

Discussion/conclusion Club drugs use among MSM overall is worryingly high locally. In particular, HIV-positive MSM use more crystal meth and GHB/GBL, and these men are more likely to engage in unprotected anal intercourse. These data are sobering and serve as a reminder that STI and drug services should work together.

\section{CHEMSEX AND THE CITY: SEXUALISED SUBSTANCE USE IN GAY BISEXUAL AND OTHER MEN WHO HAVE SEX WITH MEN}

${ }^{1}$ Ming Lee*, ${ }^{1}$ Aseel Hegazi, ${ }^{1}$ Alison Barbour, ${ }^{2}$ Bavithra Nathan, ${ }^{2}$ Simon Green, ${ }^{2}$ Richard Simms, 'Mark Pakianathan. ${ }^{1}$ The Courtyard Clinic, Wandswort Integrated Sexual Health, St George's Healthcare NHS Trust, London, UK; ${ }^{2}$ Department of Sexual Health, The Wolverton Centre, Kingston Hospital NHS Foundation Trust, London, UK

\subsection{6/sextrans-2015-052126.11}

Background/introduction Sexualised substance use (chemsex) is an emergent phenomenon amongst some gay, bisexual and other men who have sex with men (GBMSM).
Aim(s)/objectives To describe patterns of chemsex and clinical characteristics of GBMSM attending two London sexual health clinics.

Methods Retrospective case-notes review. Data on demographics, chemsex practices, sexual behaviour, STI diagnoses and HIV status extracted from a new holistic standardised profoma used in GBMSM clinics June to December 2014.

Results 27\% $(\mathrm{n}=127)$ of 531 cases disclosed drug use. 59\% $(\mathrm{n}=73 / 124)$ reported chemsex, 13\% ( $\mathrm{n}=15 / 116)$ injected. Drugs: Mephedrone ( $\mathrm{n}=48)$, GHB/GBL $(\mathrm{n}=38)$, Crystal Meth $(\mathrm{n}=28)$ and Cocaine $(\mathrm{n}=8) .1 / 3$ disclosed $>$ one chemsex session/month. Chemsex was significantly associated with the risk taking behaviours transactional sex, group sex, fisting, sharing sex toys, HIV and hepatitis sero-discordancy $(\mathrm{p}<0.05)$, more reported sexual partners (median 3 vs. 2 in past 3 months; $\mathrm{P}<0.0001$ ) and HIV positivity (35\% vs $7 \% \mathrm{p}<0.0001)$. STIs were diagnosed more frequently in chemsex participants; Gonorrhoea $(39 \%$ vs. $6 \%$ p $<0.0001)$, Chlamydia $(11 \%$ vs. $4 \%$ p $=$ $0.05)$, Hepatitis C $(5 \%$ vs $0.3 \% \mathrm{p}=0.03)$ and PEPSE was more frequently prescribed $(14 \%$ vs. $2 \% \mathrm{p}=0.001) .42 \%$ of patients perceived chemsex to have had an adverse consequences on their physical/ mental health or career.

Discussion/conclusion The majority of GBMSM reporting chemsex were HIV negative and many perceived negative consequences from chemsex. It was also significantly associated with risk taking behaviours, STIs, hepatitis $\mathrm{C}$ and being HIV positive. A holistic assessment of GBMSM enables the identification of opportunities for targeted prevention, health promotion and wellbeing interventions.

\section{ASSOCIATIONS BETWEEN REPEAT ATTENDANCES, SEXUALLY TRANSMITTED INFECTIONS AND CHILD SEXUAL EXPLOITATION IN UNDER 16 YEAR OLDS ATTENDING GENITOURINARY MEDICINE CLINICS}

${ }^{1}$ Christopher Ward*, ${ }^{2}$ Gwenda Hughes, ${ }^{2}$ Holly Mitchell, ${ }^{3}$ Karen Rogstad. ${ }^{1}$ Central Manchester University Hospitals NHS Foundation Trust, Manchester, UK; ${ }^{2}$ Public Health England, London, UK; ${ }^{3}$ Sheffield Teaching Hospitals NHS Foundation Trust, Sheffield, UK

\subsection{6/sextrans-2015-052126.12}

Background Child sexual exploitation (CSE) diagnoses are difficult to make, often with no symptoms or signs. Previous reports suggested that sexually transmitted infections (STIs) are a CSE marker but currently there is no evidence for this.

Aim To investigate associations between attendance patterns and STIs with CSE to refine clinic-based CSE risk algorithms.

Methods STI diagnoses among < 16 year-olds during 2012 were extracted from clinics using the genitourinary medicine clinic activity dataset (GUMCAD). Clinics with $>18$ STI diagnoses (all STIs) were contacted for recruitment. Cases were defined as patients with a confirmed, bacterial or protozoal STI. Controls were defined as age and gender matched asymptomatic patients at the same clinic without STIs. An online data collection tool was developed to capture additional CSE risk factors on cases and controls. A protocol was created to aid CSE definition and stratification.

Results During 2012 in England, there were 12,819 attendances of young people aged 13-15 and 2337 STIs diagnosed: 1040 (44.5\%) were chlamydia, 220 (9.4\%) gonorrhoea and 67 (2.9\%) trichomonas. Of these infections $998(75.2 \%)$ were aged 15, 57 (4.3\%) were $\leq 13$ and 1188 (89.5\%) were female. 44 clinics had $>18$ STIs in $<16$ s, and 21 were recruited to the study. 